\title{
DETERMINACIÓN DEL ANÁLISIS EXERGÉTICO EN PLANTA POTABILIZADORA RÍO CAUCA
}

\author{
DETERMINATION OF THE EXERGETIC ANALYSIS IN THE CAUCA RIVER \\ WATER TREATMENT PLANT
}

\section{Liliana Rocío Beltrán Acevedo}

Ingeniera Ambiental, Especialista en Administración Pública y Magíster en Ingeniería Ambiental

Universidad Nacional Abierta y a Distancia - ECAPMA

lilianar.beltran@unad.edu.co

\section{Sandra Viviana Villegas F.}

Ingeniera Ambiental

Universidad Nacional Abierta y a Distancia - ECAPMA

svvillegasf@unadvirtual.edu.co

\section{RESUMEN.}

En este documento se presenta la determinación del análisis exergético en la planta potabilizadora Río Cauca. El concepto de exergía se refiere a la energía que puede ser liberada de acuerdo a la diferencia de las propiedades de los elementos químicos que conforman los cuerpos de agua entre las propiedades de los elementos químicos del ambiente de referencia o del medio ambiente. Los factores naturales y la acción humana determinan la calidad de cualquier masa de agua. Además, la calidad del agua se determina comparando los directrices estándares o normatividad (dependiendo de las políticas de cada país) con las características físicas y químicas en las plantas potabilizadoras. Se encontró que la etapa de agua tratada contiene una mayor exergía química, con un porcentaje $26,23 \%$, debido que es la etapa para la producción de agua potable se necesita mayor consumo de productos y energía.

Palabras Clave: exergía química; calidad del agua; ríos.

\section{ABSTRACT.}

This document presents the determination of exergy analysis in the Rio Cauca water treatment plant. The concept of exergy refers to the energy that can be released according to the difference of the properties of the chemical elements that make up the bodies of water between the 
properties of the chemical elements of the reference environment or the environment. Natural factors and human action determine the quality of any body of water. In addition, water quality is determined by comparing the standard guidelines or regulations (depending on the policies of each country) with the physical and chemical characteristics of the water treatment plants. It was found that the treated water stage contains a greater chemical exergy, with a percentage of $26.23 \%$, because it is the stage for the production of potable water, greater consumption of products and energy is needed.

Keywords: chemical exergy; water quality; river.

\section{INTRODUCCIÓN.}

Los cuerpos de agua son dinámicos, complejos y finitos, juegan un papel fundamental para el ciclo hidrológico, la preservación de los ecosistemas, la supervivencia humana, el desarrollo y el crecimiento económico del mundo contemporáneo. A nivel mundial existe la preocupación y desafío sobre la calidad de los cuerpos de agua (carga de nutrientes y patógenos) ya que aumentará las amenazas para salud humana, el medio ambiente y el desarrollo sostenible (WWAP, 2018). El aumento de la población humana, el cambio climático, la expansión de la actividad industrial y agrícola, la deforestación y las alteraciones en el ciclo hidrológico, generan escasez, pérdida de la calidad y desastres naturales (ONU, 2014; Arana, Giraldo \& Duarte, 2017).

Estos cuerpos de agua son la materia prima para las plantas potabilizadoras de aguas. Éstas se convierten en herramientas esenciales para la vida, ya que por medio de ellas se suministra agua potable a la comunidad, población o usuario. Sin embargo, las plantas potabilizadoras están realizando grandes esfuerzos, ya que poseen diferentes problemáticas en cuanto a su materia prima (cuerpos de agua) tales como, aumento de la población, vertimientos de agua residuales e industriales, contaminantes emergentes, entre otros.

Por otro lado, una de las herramientas con que cuenta la humanidad para garantizar la disponibilidad del agua y su gestión sostenible y el saneamiento para todos, es la exergía, que, como indicador ecológico, permite la determinación, evaluación y valoración de los costos ambientales en los cuerpos de agua y en la potabilización de agua. 
La exergía se define "como la cantidad máxima de trabajo que se puede obtener cuando alguna materia se lleva a un estado de termodinámica en equilibrio con los componentes comunes del entorno natural por medio de procesos reversibles" (Szargut 1988). De acuerdo con lo anterior, la exergía está fundamentada en la primera y segunda Ley de la termodinámica e indica la energía disponible en un sistema.

Con el análisis exergético en el tratamiento de aguas tiene la concesión de comparar el recurso más infalible de su grado imposible para un análisis energético que es insensible a la calidad energética del proceso, aunque su valor es ya de por sí un valor orientativo de dicha calidad (Uche, 2013). Así mismo, con el análisis exergético, es posible calcular la eficiencia energética de la planta diseñada para cumplir los objetivos de eficacia en el proceso (estándares de calidad a la salida de dichas plantas).

\section{METODOLOGÍA.}

En este trabajo de investigación se adaptó la metodología utiliza por Lozano (2011). Para el desarrollo de esta investigación se tuvo en cuenta las definiciones de exergía, exergía química y exegía en los flujos de agua.

\section{Exergía}

El concepto de exergía se definió por primera vez por Rant 1953, el cual ha adquirido de gran interés en la actualidad en las diferentes aplicaciones en conversión de energía, optimización de procesos, diagnóstico, análisis de sistemas complejos, análisis de sostenibilidad, exercoecología, biología entre otros, (Szargurt, 1996; Oliveira, 2006; Dincer \& Ronsen 2007;, Chen, 2007, Jorgensen, 2006, Valero 2010, Uche, 2013).

Por consiguiente, se aborda las siguientes definiciones de exergía:

"La cantidad de trabajo obtenible cuando alguna materia se lleva a un estado de equilibrio termodinámico con los componentes comunes del entorno natural por medio de procesos reversibles" (Szargut, Morris, Steward, 1988). 
Otra definición según los autores (Valero \& Valero, 2010) define la exergia como "la máxima cantidad de trabajo que un sistema puede realizar llevándolo hasta el estado de equilibrio con el medio que le rodea en procesos reversibles". Además, la exergía es un "proceso que identifica los impactos al medio ambiente y permite alcanzar la eficiencia de los recursos" (Hepbasli et al., 2007).

Los autores (Dincer 2011) definen "la exergía es una medición general de la diferencia del sistema respecto a un ambiente de referencia; este ambiente de referencia actúa como un sistema infinito, es una fuente, un sumidero de calor y materiales"

\section{Exergía de los flujos de agua}

Los componentes de la exergía que conforman las condiciones físicas de un flujo de agua: térmica, mecánica, cinética, potencial y química. La exergía específica se define de la siguiente forma (Martínez, 2009):

$$
\begin{aligned}
& \boldsymbol{b}_{\boldsymbol{H} \mathbf{} \boldsymbol{O}}=\frac{\boldsymbol{C} \boldsymbol{p}_{\boldsymbol{H} \mathbf{} \boldsymbol{O}}\left[T_{a}-T_{0}-T_{0} \ln \left(\frac{T a}{T_{0}}\right) \mid\right.}{\text { Ex.Térmica t }}+\frac{V_{\mathrm{H} 2 \mathrm{O}}\left(P_{a}-P_{0}\right)}{\text { Ex.Mecánica }}+\frac{\left.\mid \sum y_{i}\left(\Delta G_{f i}+\sum n_{e} b_{c h, n e}+\sum n_{e} b_{c h, n e}\right)_{i}\right\rfloor_{p}^{+}}{\text {Ex.Química }}+ \\
& \frac{R T_{0} \sum x_{i} \ln \frac{a_{i}}{a_{0}}}{\text { Ex.Concentración }}+\frac{\frac{1}{2}\left(V_{a}^{2}-V_{0}^{2}\right)}{\text { Ex.Cinética }}+\frac{g\left(z_{a}-z_{0}\right)}{\text { Ex.Potencial }} \quad \text { (EC. 1) }
\end{aligned}
$$

Para este estudio no se tuvo en cuenta las componentes físicas para el cálculo en los flujos de agua, al no ser representativas en los procesos de potabilización, se tendrán en cuenta la composición del agua para el cálculo de la exergía: concentración y química. Además, para el cálculo de la exergía para los flujos de agua en los procesos de potabilización estarán definidas por (Lozano, 2011):

$$
\begin{aligned}
& b_{w i}=b_{M I}+b_{M O}+b_{N P}+b_{\text {arena }} \quad \text { (Ec. 2) } \\
& \mathrm{b}_{\mathrm{IM}}=\mathrm{b}_{\mathrm{H} 2 \mathrm{O}}+\mathrm{b}_{\text {sales }} \quad(\text { Ec. } 3)
\end{aligned}
$$

Donde: 
- $b_{w i}$ : Exergía química del flujo de agua i

- $b_{M I}$ : Exergía de la materia inorgánica compuesta por el agua pura y la contribución de las sales disueltas, calculada como exergía de concentración al considerarse sustancias presentes en el ambiente de referencia

- $b_{M O}$ y $b_{N P}$ : Exergía química de la materia orgánica y los compuestos de nitrógeno y fósforo presentes en el agua

- $b_{\text {arena: }}$ Exergía química de la arena

Para Lozano (2011) para el cálculo de la exergía del agua pura ( $\mathrm{b}_{\mathrm{H} 2 \mathrm{O}}$ ) como de las sales, se utiliza es la de concentración (Ec.4), en la que $x_{i}$ hace referencia a la concentración molar de cada elemento en la mezcla, $a_{i}$ representa la actividad de la especie en la mezcla y $a_{0}$ la actividad de la especie en el ambiente de referencia.

$$
b_{c h, c}=R T_{0} \Sigma x i \ln \left(\frac{a_{i}}{a_{0}}\right)(\mathrm{Ec} .4)
$$

\section{Exergía química para una mezcla}

Un afluente, lleva una variedad de componentes transformándose en una mezcla, es así que la exergía química en este caso, relaciona el coeficiente de actividad de la sustancia en el agua (al), y se calcula con la ecuación 5 , por lo cual se requiere determinar la tendencia de la sustancia a solicitar una fase y la carga iónica (Bastidas, 2014).

$$
\begin{aligned}
& \text { Bch }=\mathrm{Q} *(\text { } x i B i+\mathrm{RTo} \Sigma x i L n a i)(\text { Ec. } 5) \\
& \mathbf{a i}=\Upsilon \mathrm{i} \times \text { mi }(\text { Ec } 6) \\
& B c h=\text { Exergía química } \\
& \mathbf{Q}=\text { Caudal }(\mathrm{L} / \mathrm{s}) \\
& x i=\text { Fracción molar } \\
& \mathbf{B i}=\text { Exergía química estándar del elemento }(\mathrm{kJ} / \mathrm{mol}) \\
& \mathbf{R}=\text { Constante de los gases }(\mathrm{J} / \mathrm{mol} \mathrm{K}) \\
& \text { To }=\text { Temperatura }(\mathrm{K}) \\
& a_{\mathbf{i}}=\text { Coeficiente de actividad de la sustancia en el agua } \\
& \Upsilon_{i}=\text { Fugacidad } \\
& \mathbf{m}_{\mathbf{i}}=\text { Molalidad }
\end{aligned}
$$




\section{Planta de Potabilización Río Cauca}

La Planta de potabilización Río Cauca, está localizada en la ciudad de Cali, fue construida por la empresa EMCALI, en el año de 1953, se iniciaron los trámites para la ampliación de la capacidad de agua potable, por el crecimiento y demanda de la población. Esta planta tiene una capacidad de $2 \mathrm{~m}^{3} / \mathrm{s}$.

Esta es la descripción de cada uno de los procesos de producción de la planta de potabilización Río Cauca de acuerdo con el Sistema de Información de Agua Potable (SIDAP):

Captación y Bocatoma: Su captación es lateral y permite la eliminación de sólidos gruesos y flotantes.

Desarenador y equipo de remoción de basuras y arena: este paso consiste tamizar el agua por medio de rejillas con el fin de remover las basuras que han estado al sistema y en la extracción de lodos y arenas, mediante hidroeyectores y una bomba sumergible, la cual actúa como una aspiradora adaptada en un soporte del puente desarenador.

El desarenador tiene como función principal es permitir la sedimentación de las partículas más pequeñas (aproximadamente de tamaño 0,2 mm) que no han sido removidas por las rejillas de operación manual.

Estación de Bombeo e impulsión de agua cruda: El proceso de bombeo e impulsión de agua cruda desde el pozo de succión hasta la Planta Río Cauca, así como las condiciones eléctricas necesarias para la operación inicial y normal de la Bocatoma.

\section{Cámara de Mezcla Rápida y Distribución de Caudales}

El agua cruda viene por impulsión por medio de dos tuberías, descargan a una cámara de mezcla en la cual se aplica un coagulante de alambre, la dosificación del coagulante se realiza por medio de 5 bombas dosificadoras, las cuales transportan el coagulante mediante dos tuberías de PVC.

\section{Área De Clarificadores}

En la Planta Río Cauca los procesos de floculación y sedimentación se realizan en clarificadores de flujo vertical. 


\section{RESULTADOS.}

Se promediaron a un año los cálculos de exergía y las concentraciones de solutos se convirtieron a unidades moles $\mathrm{L}^{-1}$.

En la Tabla 1 se presentan el caudal de entrada y salida en la planta potabilizadora.

Tabla 1. Caudal de agua de entrada, salida de la planta Río Cauca

\begin{tabular}{|l|l|l|}
\hline Planta & $\begin{array}{l}\text { Caudal de entrada } \\
\left(\mathbf{L ~ s}^{-\mathbf{1}} \mathbf{)}\right.\end{array}$ & $\begin{array}{l}\text { Caudal de salida } \\
(\mathbf{L ~ s} \mathbf{~} \mathbf{~}\end{array}$ \\
\hline Río Cauca & $2.352,8$ & $2.255,08$ \\
\hline
\end{tabular}

En la Tabla 2 se muestran los datos físico-químicos promediado para el año seleccionado de la planta potabilizadora Río Cauca.

Tabla 2. Parámetros físicos - químico anual de la planta Río Cauca

\begin{tabular}{|c|c|r|r|r|r|}
\hline & & \multicolumn{3}{|c|}{ Concentración promedio } \\
\hline Parámetro & Unidad & $\begin{array}{c}\text { Agua } \\
\text { cruda }\end{array}$ & $\begin{array}{c}\text { Agua } \\
\text { clarificad } \\
\text { a }\end{array}$ & $\begin{array}{c}\text { Agua } \\
\text { filtrada }\end{array}$ & $\begin{array}{c}\text { Agua } \\
\text { tratada }\end{array}$ \\
\hline Cloro residual libre & $\mathrm{mg} / \mathrm{L} \mathrm{Cl}_{2}$ & - & 0,42 & 0,15 & 1,64 \\
\hline Aluminio residual & $\mathrm{mg} / \mathrm{L} \mathrm{Al}_{2}$ & 0,04 & 0,07 & 0,03 & 0,03 \\
\hline Hierro total & $\mathrm{mg} / \mathrm{L} \mathrm{Fe}^{-}$ & 4,79 & 0,08 & 0,04 & 0,04 \\
\hline Cloruros & $\mathrm{mg} / \mathrm{L} \mathrm{Cl}^{-1}$ & 5,35 & 11,25 & 11,08 & 12,76 \\
\hline Alcalinidad total & $\mathrm{mg} / \mathrm{L} \mathrm{CaCO}_{3}$ & 24,98 & 18,70 & 18,38 & 22,45 \\
\hline Dureza total & $\mathrm{mg} / \mathrm{L} \mathrm{CaCO}_{3}$ & 40,97 & 41,48 & 42,05 & 46,61 \\
\hline Calcio & $\mathrm{mg} / \mathrm{L} \mathrm{Ca}^{+2}$ & 8,31 & 8,13 & 9,02 & 9,79 \\
\hline Magnesio & $\mathrm{mg} / \mathrm{L} \mathrm{Mg}^{+2}$ & 4,92 & 5,15 & 4,75 & 5,39 \\
\hline Sulfatos & $\mathrm{mg} / \mathrm{L} \mathrm{SO}^{-2}$ & 20,34 & 17,77 & 17,73 & 17,68 \\
\hline Nitratos & $\mathrm{mg} / \mathrm{L} \mathrm{NO}_{3}{ }^{-1}$ & 2,52 & & & 2,66 \\
\hline Nitritos & $\mathrm{mg} / \mathrm{L} \mathrm{NO}_{2}{ }^{-1}$ & 0,07 & & & 0,01 \\
\hline Manganeso & $\mathrm{mg} / \mathrm{L} \mathrm{Mn}^{+7}$ & 0,87 & & & 0,03 \\
\hline Fosfatos & $\mathrm{mg} / \mathrm{L} \mathrm{PO}_{4}^{-3}$ & 0,19 & & & 0,03 \\
\hline Turbidez & $\mathrm{NTU}$ & 65,80 & & & 0,63 \\
\hline
\end{tabular}


Se realizó la variación exergética de los procesos del agua cruda, clarificada, filtrada y tratada, se presenta la exergía química en $\mathrm{KJ} / \mathrm{L}$ de los componentes estudiados.

Tabla 3. Exergía Química

\begin{tabular}{|c|c|}
\hline Componente & Exergía química KJ/L \\
\hline Cruda & 0,319627234 \\
\hline Clarificada & 0,327988594 \\
\hline Filtrada & 0,335922315 \\
\hline Tratada & 0,369905112 \\
\hline
\end{tabular}

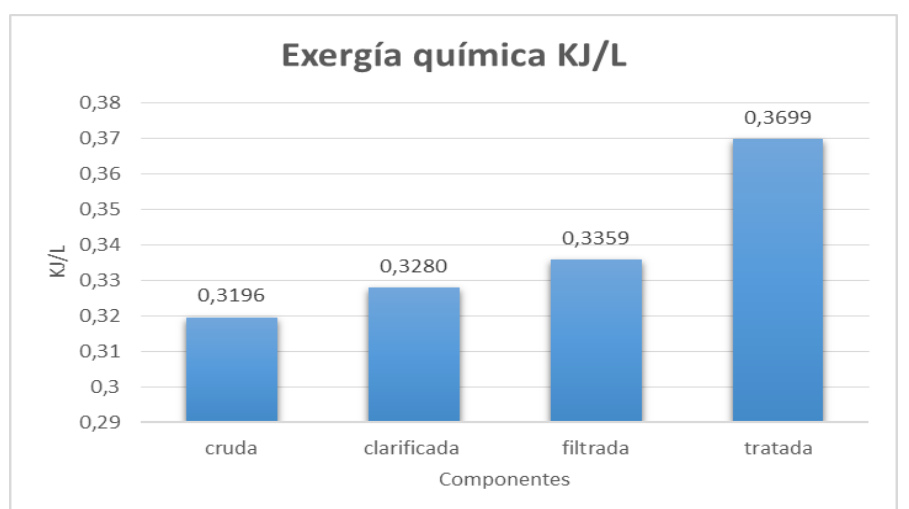

Gráfica 1. Exergía Química en los procesos de potabilización Río Cauca

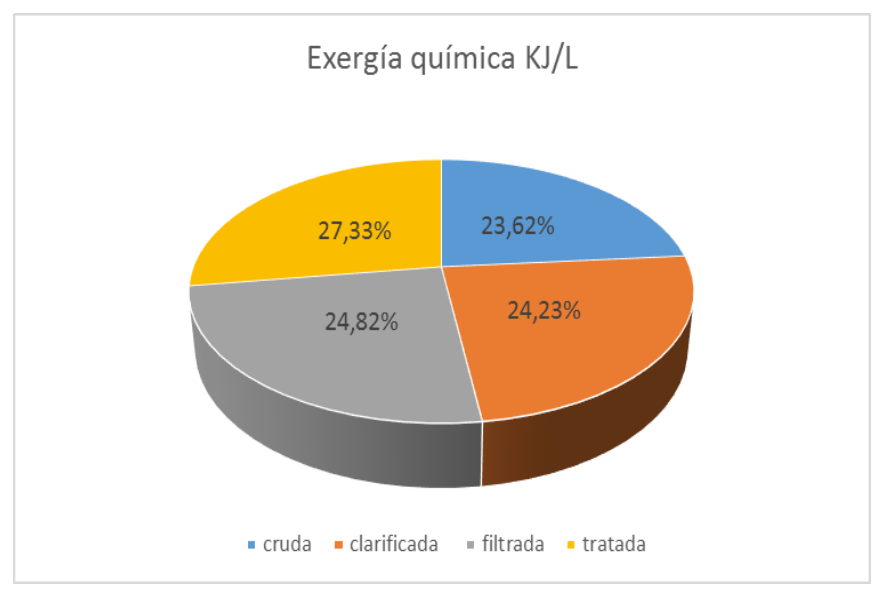

Gráfica 2. Porcentaje de la exergía química en los procesos de potabilización Río Cauca 
En las gráficas 1 y 2 se muestra que el mayor consumo fue en la etapa de agua tratada, dado que se requiere mayor energía, esfuerzo y productos para poder otorgar un agua para el consumo humano.

En cuanto a los Vatios utilizados en cada componente se establece lo siguiente:

Tabla 4. Análisis de KW

\begin{tabular}{|c|c|}
\hline Componente & KW \\
\hline Cruda & 762,457983 \\
\hline Clarificada & 782,403671 \\
\hline Filtrada & 801,329246 \\
\hline Tratada & 834,165619 \\
\hline
\end{tabular}

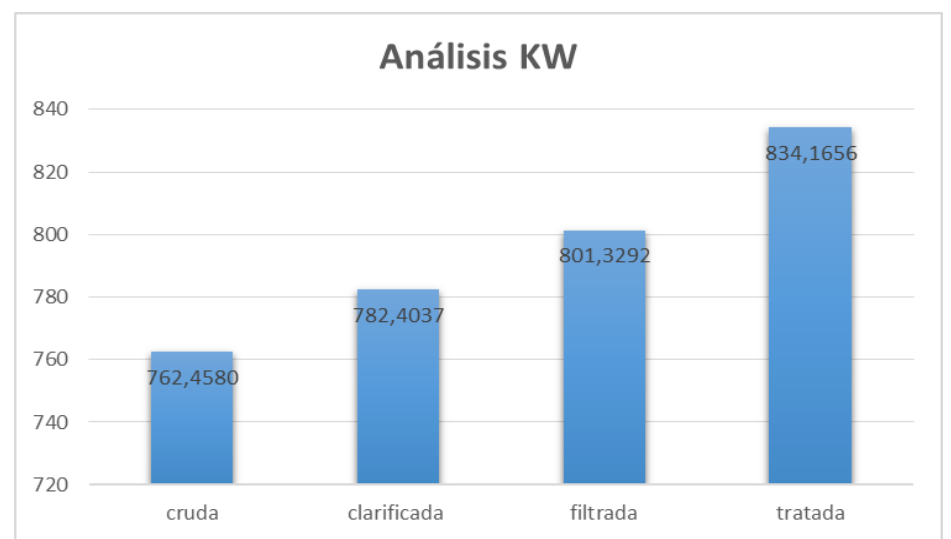

Gráfica 3. Análisis de KW

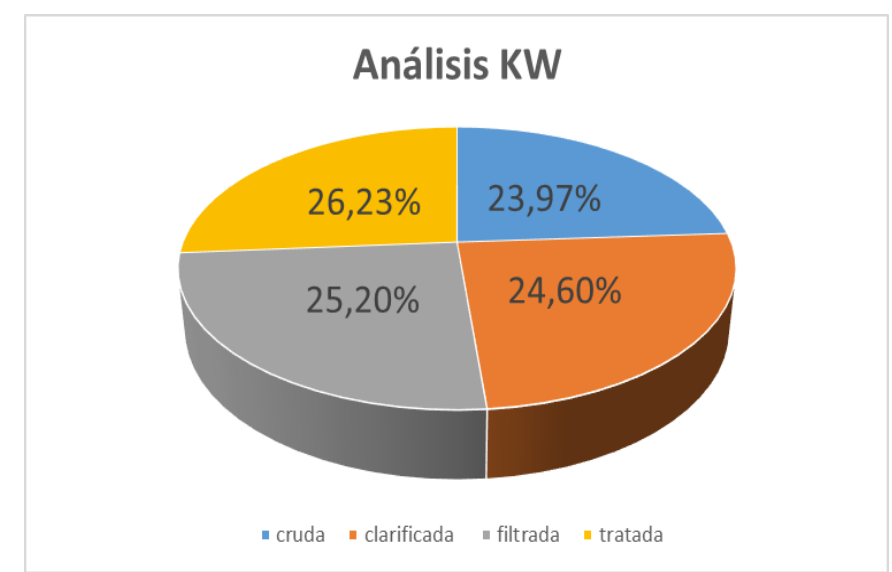

Gráfica 1. Porcentaje de KW 
En las gráficas 3 y 4 se visualiza, que en la etapa de agua tratada hay un incremento en el consumo de $\mathrm{KW}$, y al mismo tiempo una mayor cantidad de energía para que los equipos puedan mejorar las condiciones del agua, por otro lado, esta es la etapa final donde se les otorga a los caleños un agua que cumple con la normatividad vigente y que es apta para su consumo.

\section{CONCLUSIONES.}

El análisis exergético permite comprender los impactos de la calidad del agua de la fuente estudiada, por otro lado, admite a proceder chequeos los cuales acepta o corrobora los resultados del cálculo de la exergía química.

El concepto de exergía sigue teniendo auge en las últimas décadas ya que permite analizar y comparar las etapas ineficientes del proceso, con el fin de identificar las oportunidades de mejoras en el sistema de tratamiento de potabilización.

\section{BIBLIOGRAFÍA.}

Arana-Gutiérrez, A.,Giraldo-, Alzate, O. \& Duarte, L. (2017). Las guerras globales por el oro azul en el siglo XXI. (14) 1, 154-168. Recuperado de https://revistas.unilibre.edu.co/index.php/criteriojuridico/article/view/16 $\underline{29 / 1205}$

Bastidas, M. (2014). Evaluación exergética de la calidad del agua de tres ríos en Colombia. Recuperado de http://www.bdigital.unal.edu.co/47036/1/34561303-Mary.pdf

Chen, G.Q \& Xi Ji (2007). Chemical exergy based evaluation of water quality. Ecological Modelling.

Lozano A., (2011). Análisis exergético en plantas de tratamiento en el ciclo integral del agua. Recuperado de https://zaguan.unizar.es/record/6501? In=es

Ministerio de Ambiente, Vivienda y Desarrollo Territorial. Decreto 3930 de 2010 Recuperado de 
http://www.minambiente.gov.co/images/normativa/decretos/2010/dec 3930 2010.pdf

Moreno, T. (2011). Aplicación de la exergía para la definición de indicadores del aprovechamiento del agua. Recuperado de http://zaguan.unizar.es/record/5956/files/TAZ-PFC-2011-275.pdf

Morris, D.R., Szargut, J., 1986. Standard chemical exergy of some elements and compounds on the planet earth. Energy 11 (8), 733-755.

Organización Naciones Unidas (2014). Decenio Internacional para la Acción "El agua fuente de vida" 2005-2015. Recuperado de http://www.un.org/spanish/waterforlifedecade/quality.shtml

Szargut, J., Morris, D. R., Steward, F. R. (1988). Exergy analysis of thermal, Chemical, and metallurgical processes. New York: Hemisphere.

Riekert, L. (1974). Chem. Eng. Sci. 29, 1613.

World Water Assessment Programme (WWAP) (2015). Water for a Sustainable World. United Nations Educational, Scientific and Cultural Organization UNESCO. Recuperado de http://www.unesco.org/new/es/naturalsciences/environment/water/wwap/wwdr/

El presidente de la República de Colombia. Decreto 1594 de 1984. Usos del agua y residuos líquidos. Recuperado de http://www.ideam.gov.co/documents/24024/36843/Dec 1594 1984.pd f/aacbcd5d-fed8-4273-9db7-221d291b657f

Zaleta-Aguilar, A., Ranz, L., Valero, A., 1998. Towards a unified measure of renewable resources availability: the exergy method applied to the water of a river. Energy Conversion Manag. 39, 1911-1917. 